\title{
Entwicklung von Praxiskompetenz durch Kooperationsprozesse von Lehrkräften
}

\author{
Thiemo Bloh
}

Eingegangen: 14. Juni 2021 / Überarbeitet: 29. Oktober 2021 / Angenommen: 3. November 2021 / Online publiziert: 25. November 2021

(C) Der/die Autor(en) 2021

Zusammenfassung Lehrkräftekooperation wird generell eine positive Bedeutung in Bezug auf Schul- und Unterrichtsentwicklung zugeschrieben. Dabei sind empirische Belege für eine positive Wirksamkeit nach wie vor kaum vorhanden, es gibt sogar Befunde zu ,negativen“ Konsequenzen von Lehrkräftekooperation. Um diese Widersprüchlichkeit zu klären, wurde in der vorliegenden Arbeit Kooperation nicht als Instrument bzw. als Technik betrachtet, sondern als soziale Praxis verstanden, in der eigenlogisches, kollektiv-implizites Wissen (re)produziert wird (Community of Practice). Parallel dazu wurde ein praxeologisches Kompetenzverständnis (Praxiskompetenz) eingeführt, das wesentlich auf die Praxeologie Pierre Bourdieus zurückgeht und den Zusammenhang zwischen Lehrkräftekooperation als Community of Practice und kollektiv strukturierter, individueller Kompetenz theoretisch verdeutlicht. Die empirischen Befunde, welche mittels der Dokumentarischen Methode generiert wurden, verweisen auf die Bedeutung unterschiedlicher Relationslogiken (Nicht-Passung, Entfaltung, Herausforderung) für das ,Lernen' von oder innerhalb von Praxiskompetenz(en) und damit auch auf die Wichtigkeit einer grundlegend kollektiv gerahmten Perspektive auf Lehrkräftekooperation. Vor diesem Hintergrund ist ein allzu positiver Blick auf Lehrkräftekooperationsprozesse kritisch zu betrachten.

Schlüsselwörter Lehrkräftekooperation · Community of Practice · Handlungsorientierung · Praxiskompetenz · Dokumentarische Methode

Dr. Thiemo Bloh $(\bowtie)$

Universität Paderborn, Warburger Straße 100, 33098 Paderborn, Deutschland

E-Mail: bloh@upb.de 


\title{
Development of practical competence through teacher collaboration
}

\begin{abstract}
Teacher collaboration is generally attributed to positive influence on school development and teacher professionalization. But empirical evidence of a positive effect is still hardly found; there are even findings which refer to negative consequences of teacher collaboration. Teacher collaboration should not be seen as an instrument or a technique, but rather as a social practice, which refers to (re)production of collective-implicit knowledge with a logic of its own (Community of Practice). In parallel a practical understanding of competence is introduced (practical competence), which is built on the Praxeologie of Pierre Bourdieu and can clarify the connection of teacher collaboration as a Community of Practice on the one hand, and collective structured, yet individual competence on the other hand. The empirical findings, which are generated with the documentary method, show the importance of different logics of relations (non-fitting, unfolding, challenging) for learning of or within practice competence(s) and therefore also the importance of a fundamentally collective perspective on teacher collaboration. Against this backdrop a mostly positive view on teacher collaboration should be seen rather critical.
\end{abstract}

Keywords Teacher collaboration - Community of Practice - Action orientation · Practical competence $\cdot$ Documentary method

\section{Einleitung}

Lehrkräftekooperation wird häufig als Instrument verstanden, das Lehrkraftkompetenz und Schulentwicklung positiv beeinflusst (Fussangel und Gräsel 2014). Betrachtet man jedoch die Befundlage, so stellt sich diese allgemein durchaus unterschiedlich bis gar widersprüchlich dar (Fussangel und Gräsel 2014; Trumpa et al. 2016; Vangrieken et al. 2015). Die (positive) Wirksamkeit von Lehrkräftekooperation ist nach wie vor empirisch kaum belegt, Trumpa et al. (2016, S. 84) sprechen von einem ,„persistierende[n] Erkenntnisdefizit“. Zudem lassen sich Arbeiten finden, die von negativen Effekten berichten (Achinstein 2002; Horn 2015). ,,Teacher collaboration should not be seen as a magic solution that solves all problems as it can entail negative consequences" (Vangrieken et al. 2015, S. 36).

Die Frage, die sich dabei stellt, ist, wie ein Konstrukt, mit dem große Hoffnungen auf Schul- und Kompetenzentwicklung der beteiligten Lehrkräfte durchaus berechtigterweise verknüpft sind - denn die theoretisch wie empirisch positiven Befunde (bspw. Bonsen und Rolff 2006; Halbheer und Kunz 2009; Kullmann 2009; Louis et al. 1996) bleiben ja bestehen - gleichzeitig auch negative Konsequenzen haben kann. Die (mögliche) Erklärung, welche in diesem Beitrag gegeben wird, verschiebt die Perspektive auf Lehrkräftekooperation und der sich daraus entwickelnden Kompetenz der Lehrkräfte, indem systematisch die Praxeologie Pierre Bourdieus (Bourdieu 1979) auf den Gegenstand Lehrkräftekooperation angewandt und diese somit als soziale Praxis verstanden und von einem technischen Kooperationsverständnis differenziert wird (Abschn. 2). Der zu dieser Konzeption passende Kompetenzbegriff wird in Abgrenzung derzeit dominanter (Pfadenhauer 2010) erziehungswis- 
senschaftlich-psychologischer Kompetenzmodelle (bspw. Klieme und Hartig 2007) entwickelt und baut wesentlich auf dem Konzept der „Handlungsorientierung“ (Asbrand 2008; Nohl et al. 2015) auf (Abschn. 3). Die empirischen Befunde, welche mit der Dokumentarischen Methode (Bohnsack 2008, 2017; Nohl 2017; Przyborski 2004) (Abschn. 4) ausgewertet wurden, basieren auf videographierten Kooperationssitzungen sowie berufs-biographisch narrativen Interviews mit den beteiligten Lehrerinnen und verweisen auf die Bedeutung einer praxeologischen Perspektive auf Lehrkräftekooperation sowie auf das Potenzial des Konstrukts der Praxiskompetenz (Abschn. 5).

\section{Technisches und praktisches Kooperationsverständnis}

Entsprechend der positiven Stimmungslage zu Lehrkräftekooperation gibt es nicht nur ein breites Spektrum an Arbeiten, die sich dem Konstrukt Lehrkräftekooperation und möglichen Folgen widmen (vgl. Bloh 2021, S. 14), sondern auch einige Überblicksartikel und Metaanalysen, welche Arbeiten zusammenfassen und ordnen (Fussangel und Gräsel 2014; Lomos et al. 2011; Vescio et al. 2008). Jenseits konkreter thematischer Zuordnungen lassen sich Ansätze zu Lehrkräftekooperation m.E. vor allem zwei grundlegenden Ideen zuordnen:

- einem technischen Kooperationsverständnis, zu welchem m.E. auch die viel zitierten Ansätze von Bonsen und Rolff (2006), Steinert et al. (2006) und Gräsel et al. (2006) zählen, sowie

- einem praktischen Kooperationsverständnis, welches sich eher im anglo-amerikanischen Raum finden lässt (Grossmann et al. 2001; Horn und Little 2010; Horn 2015).

Diese Differenzierung ist deshalb bedeutsam, da sie nicht nur auf eine grundlegend andere Idee und entsprechend auch auf grundlegend andere Konsequenzen in Bezug auf Lehrkräftekooperation verweist, sondern auch einen ersten Schritt zur Erklärung der einleitend erwähnten widersprüchlichen Befunde darstellen kann.

\subsection{Zu einem technischen Kooperationsverständnis}

Lehrkräftekooperation wird in einem technischen Verständnis als etwas konstruiert, das herzustellen ist (bspw. Rolff 2015), für das Zeit und Räume bereitgestellt werden müssen (bspw. Richter und Pant 2016), kurz, das man tun oder lassen kann. Damit einher schwingt entsprechend immer die Annahme, dass man Lehrkräftekooperation durch Bereitstellung struktureller Rahmenbedingungen herstellen könnte. Lehrkräftekooperation wird hier als ,Instrument' (bspw. Fussangel 2008; Kansteiner et al. 2020) gesehen und Kooperation ist eine Technik, die, richtig angeregt und angewandt, den erhofften Fortschritt (bspw. Kompetenzsteigerung) bringt oder zumindest begünstigt.

In einer solchen Perspektive werden jedoch unterschiedliche soziale Praxen, welche Kooperation rahmen, nicht berücksichtigt, sondern unter dem Oberbegriff ,Lehrkräftekooperation' subsumiert und egalisiert. Denn so ist bspw. mit der Tatsache, 
dass kooperiert wird, wie häufig (bspw. Richter und Pant 2016) und mit wem (bspw. Järvinen et al. 2012) dies geschieht, oder auch welche Form (bspw. Gräsel et al. 2006) oder welches Niveau (bspw. Steinert et al. 2006) bedient wird, nichts über die praktische Rahmung der Interaktion gesagt. Während Kollegium A eventuell darüber spricht, wie man den besten Unterricht gestaltet, kooperiert Kollegium B, wie man mit möglichst wenig eigenem Arbeitsaufwand den Unterricht gestalten kann beide planen, aus theoretisch-abstrakter Perspektive, jedoch kooperativ Unterricht, sogar, guten ' Unterricht aus ihrer Perspektive. Ob in beiden Varianten jedoch gleichwertiger Unterricht als Produkt erzeugt wird, wäre eine andere, letztlich empirische Frage. Und eine Möglichkeit, diese Frage theoretisch zu rahmen, bietet die Praxeologie Bourdieus bzw. ein darauf aufbauendes praktisches Kooperationsverständnis.

\subsection{Zu einem praktischen Kooperationsverständnis}

$\mathrm{Zu}$ einem praktischen Kooperationsverständnis zählen bspw. die Arbeiten der Arbeitsgruppe um Little (Horn 2005, 2015; Horn und Little 2010), die sich vorrangig mit den Effekten von Communities auf Schule, Lehrkräfte und Schülerinnen und Schüler befasst. Dabei wird u.a. auch auf das Konzept der Community of Practice (CoP) (Lave und Wenger 1991) verwiesen, welches zwar nicht explizit auf Lehrkräftekooperation bezogen ist, allerdings von einigen Autoren (Bloh und Bloh 2016, 2020; Hodkinson und Hodkinson 2004; Horn 2005) in dieser Hinsicht heuristisch brauchbar gemacht wurde. Vor allem in seiner ursprünglichen Form (Lave und Wenger 1991) ist das Konzept der Community of Practice - wissenssoziologisch interpretiert - für die Analyse von Lehrkräftekooperationsprozessen fruchtbar. Im Kontext des Konstrukts CoP stellt sich nicht die Frage, wie diese implementiert und nutzbar gemacht werden können, sondern eher welche Effekte hiermit verbunden sind. „The research task is not to see whether they exist or not, but to identify their characteristics in relation to learning" (Hodkinson und Hodkinson 2004, S. 29). Somit wird in einem praktischen Kooperationsverständnis auch die Idee eines (steuerbaren) Instruments verlassen und die Idee einer eigenlogisch-sozialen Praxis zentral gestellt.

CoPs zeichnen sich allgemein durch verbindendes, kollektiv-implizites Wissen aus (bspw. Vorstellungen zu gutem Unterricht). Dieses Wissen ist in Praktiken (bspw. eine Art und Weise zu Unterrichten), Artefakte (bspw. Unterrichtsmaterial) und Diskursen (bspw. Unterrichtsplanungen, in der auch Vorstellungen über guten Unterricht einfließen, ohne explizit thematisiert zu werden) eingeschrieben (Lave und Wenger 1991, S. $105 \mathrm{ff}$.). Es geht also weniger darum was getan bzw. worüber gesprochen wird, sondern - in Anlehnung an Bohnsack (2008) und die Grundprinzipien der Dokumentarischen Methode (s. unten) - wie über das gesprochen wird, worüber gesprochen wird, in welchem Rahmen die Handlung stattfindet. Damit sind nun nicht abstrakte Interaktionsformen gemeint, sondern ein „Orientierungsrahmen“ (Bohnsack 2011, 2017), welchen Bohnsack (2011) synonym zum „Habitus“ (Bourdieu 1979) begreift, also „Wahrnehmungs-, Bewertungs- und Handlungsschemata“ (Bourdieu und Wacquant 2013, S. 37), durch welche Praxis und Kompetenz konstituiert werden (siehe genauer in Abschn. 3.3) und welche in einer CoP gelernt werden (können). Entsprechend ist Lernen nicht einfach ,Wissenserwerb“, sondern 
Teilhabe an einer sozialen Praxis und der „Erwerb der in dieser Gruppe gültigen Denkweisen und Problemlösemechanismen“ (Gruber 1999, S. 165). Kompetenz ist damit „historically and socially defined“ (Wenger 2000, S. 226).

Begreift man, wie Hodkinson und Hodkinson (2004), eine CoP als Feld (vgl. dazu ausführlich Bloh 2021, S. 72 ff.), also als ein Spiel, das ,viel fließender und komplexer ist als jedes nur denkbare Spiel“" (Bourdieu und Wacquant 2013, S. 135), dann sind es die in diesem Spiel geltenden Regeln, welche die Bedeutungen von Handlungen festlegen (Bourdieu und Wacquant 2013, S. 127). Die Regeln sagen also nicht, was getan werden muss, sondern was gewisse Taten bedeuten (Bourdieu 1992, S. $81 \mathrm{ff}$.) und geben damit Bedeutungsstrukturen vor, die von den Akteurinnen und Akteuren inkorporiert werden (können), was der Fähigkeit, letztlich der Kompetenz zum Mitspielen entspricht. Kompetenz entwickelt sich und wirkt also relational zum Feld bzw. zur CoP. ,Wir gehören zu einer Gruppe nicht bloß, weil wir in sie hinein geboren sind, nicht nur, weil wir behaupten, zu ihr zu gehören, noch schließlich, weil wir ihr unsere Loyalität und Anhänglichkeit schenken, sondern hauptsächlich weil wir die Welt und bestimmte Dinge in der Welt so wie sie sehen, d.h. durch die Sinndeutungen der fraglichen Gruppe hindurch“" (Mannheim 1995, S. 20). Das Feld (die CoP) bestimmt somit letztlich, welche Spielstile, welche Handlungen anerkannt werden und somit auch, was ,kompetent" ist und was nicht (Alkemeyer und Buschmann 2017) - und strukturiert darüber die Bedeutungs-, Wahrnehmungs- und Handlungsschemata der Akteurinnen und Akteure.

Im Feld existiert demnach eine ,implizite[] Pädagogik“ (Bourdieu 1979, S. 200), die dazu führt, dass die Akteurinnen und Akteure lernen, die soziale Logik des Feldes anzuerkennen, zu inkorporieren - oder eben nicht, was aber nur um den Preis des Spielausschlusses funktioniert (bspw. ,Wegbewerben', wie es von manchen Lehrkräften genannt wird). Ein Spiel mitspielen zu können, stellt eine Kompetenz dar, Spielen zu lernen, ist Kompetenzentwicklung.

\section{Praxiskompetenz}

Fällt der Begriff ,Kompetenz، wird dieser häufig nicht wie in diesem Artikel bisher gebraucht, sondern mit dem Namen Weinert (Weinert 2001) und den darauf aufbauenden Arbeiten (bspw. Klieme und Hartig 2007) verbunden. An dieser Stelle soll auf diesen Begriff nicht genauer eingegangen, sondern vornehmlich auf Probleme verwiesen werden, die dieser erziehungswissenschaftlich-psychologische Kompetenzbegriff - so funktional und wertvoll er in anderen Bereichen auch sein mag dann bekommt, wenn er auf Lehrkräftekooperation als CoP, also als soziale Praxis, angewandt wird. Eine Abgrenzung, aber auch ein Verweis auf Gemeinsamkeiten, ist notwendig, um die Konturen einer praxeologischen Perspektive auf Kompetenz (bspw. Martens 2010; Windeler 2014) zu schärfen und gleichzeitig fruchtbar innerhalb eines bestehenden Kompetenzdiskurses zu verorten. 


\subsection{Gemeinsamkeiten}

Grundlegend für den Begriff der Kompetenz nach Weinert ist zunächst die u.a. auf Chomsky zurückgehende Differenz zwischen Kompetenz und Performanz (Chomsky 1992). Chomsky unterscheidet zwischen (Sprach)Kompetenz als generativem Prinzip und der (Sprach)Performanz, also der konkreten Anwendung (Chomsky 1992, S. 14). Der entscheidende Gedanke ist, dass durch die Sprachdisposition (Kompetenz), die eine (begrenzte) Anzahl an Wörtern, Regeln, etc. beschreibt, unendlich viele Sätze (Performanz), also auch solche, die vorher noch nie gesprochen wurden, entwickelt bzw. generiert werden können. In diesem Sinne ist Kompetenz ein generatives Prinzip.

Im Gegensatz zu Chomsky (1992), der die Sprachkompetenz als zumindest zum Teil angeboren betrachtet hat (Krais und Gebauer 2014, S. 31), werden Kompetenzen nach Weinert (und auch praxeologisch) jedoch prinzipiell als erlernbar konzeptualisiert. Lernbarkeit ${ }^{1}$ (1) ist auch eine von drei Komponenten, die nach Klieme und Hartig (2007, S. 17) jede Kompetenzdefinition beinhalten muss. Daneben sind Kompetenzen kontext- bzw. bereichsspezifisch (2) und sie sind funktional (3), das heißt die „Frage ,kompetent wofür?‘ ist notwendiger Bestandteil jeder Kompetenzdefinition“. Eine praxeologische Kompetenzperspektive - die hier als Praxiskompetenz bezeichnet wird - wird diesen Kriterien gerecht und erlaubt es gleichzeitig Kompetenzen grundlegend anders zu fassen, da einige Differenzen zum ,klassischen“ Kompetenzbegriff vorliegen.

\subsection{Differenzen}

Greift man die oben ausgeführte Konzeptualisierung von Lehrkräftekooperation als soziale Praxis, also als CoP auf, dann stehen dort zwei ,Wissensformen' (vgl. generell zu Wissensformen auch Eraut 2000) im Vordergrund - das kollektive sowie das implizite Wissen. Beides ist im Kontext des Weinertschen Kompetenzbegriffs jedoch problematisch.

Denn hier werden funktional-pragmatische Kompetenzen als „Einheit von Wissen und Können“, also als ,,deklaratives und prozedurales Wissen aus einem jeweils umschriebenen Gegenstandsbereich“ (Wilhelm und Nickolaus 2013, S. 25) bezeichnet. Dabei ist das Verhältnis von deklarativem, also explizitem, und prozeduralem, also implizitem Wissen, jedoch stets ein gerichtetes. Ausgehend von deklarativem Wissen, das per Instruktion gelehrt werden kann, wird durch Erfahrung, also durch Prozeduralisierung von deklarativem Wissen, prozedurales Wissen erzeugt.

Das Problem solcher „Prozeduralisierungsmodelle“ (Neuweg 2001, S. 7) ist, dass hier implizites Wissen lediglich ,,als der nur durch Erfahrung erwerbbare Anteil oder Reifegrad von Kompetenz [erscheint; T.B.], als das, was dem ,Theoretiker' fehlt und hinzutreten muß, um explizites Wissen ,zum Laufen“ zu bringen“ (Neuweg 2001, S. 6). Allerdings können Kompetenzmodelle, die von einem solchen Prozeduralisierungsgedanken ausgehen, den Kompetenzerwerb nicht umfassend abbilden, ,weil

\footnotetext{
1 Wobei im Kontext des Kompetenzbegriffs nach Weinert mit Lernbarkeit meist auch Lehrbarkeit im Sinne einer Instruktion gemeint ist (Neumann 2013, S. 36).
} 
wir viele Kompetenzen, ja vielleicht die meisten, erwerben, ohne jemals sprachliche Instruktion erhalten zu haben“ (Neuweg 2001, S. 7).

Der entscheidende Gedanke im Konstrukt der Praxiskompetenz ist, implizites Wissen nicht als (erfahrungsbasiertes) prozeduralisiertes deklaratives Wissen zu denken, sondern als ein eigenständiges Wissen, das immer implizit bleibt, niemals explizit war und auch ,,implizit“ erworben wird“ (Polanyi 2016, S. 16). So ist Sprache bspw. regelgeleitet, ohne dass diese Regeln notwendigerweise benannt werden können (Neuweg 2001, S. 5) und auch ohne, dass die theoretische Benennung der Regel ein praktisches Beherrschen der Sprache bedingt. Sprachkompetenz verweist auf unser Vermögen, ,eine unbegrenzte Zahl von Sätzen als grammatikalisch richtig zu erkennen oder solche Sätze hervorzubringen“ (Neuweg 2001, S. 5) und nicht darauf, diese Regeln zu explizieren oder explizit anwenden zu können. Zudem, und das ist entscheidend, funktioniert das Lernen grammatikalisch korrekter Sprache nicht, sicherlich aber nicht ausschließlich, über (sprachliche) Instruktion. Die Regeln wurden also nicht explizit erlernt und dann prozeduralisiert (wodurch sie unter Umständen ,vergessen “ werden). (Mutter)Sprachkompetenzerwerb wäre dann nur schwer mit Prozeduralisierungsmodellen in Verbindung zu bringen. ,Korrekt ${ }^{\text {* }}$ Sprechen-Können ist also von der Fähigkeit der Explikation der Regeln ,korrekter Sprache zu trennen.

Greift man die Idee einer ,korrekten' Sprache auf, lässt sich daran auch die Differenz zu einem individual geprägten Kompetenzbegriff, ,which is the usual psychological perspective“ (Weinert 2001, S. 51), verdeutlichen. Die ,Anforderungen“ werden hier aus der Aufgabe gedacht, und die Kompetenz auf Seiten des Individuums verortet (bspw. korrekt sprechen können). Letztlich erinnert dieses Konstrukt der Kompetenz an das technische Verständnis von Lehrkräftekooperation. Es geht hier um (sozial-dekontextualisiertes) Wissen und Können (bspw. ,Sprache'), das das Individuum (per Instruktion) erwirbt und in verschiedenen (und möglichst vielen) Situationen anwenden, die aus der Situation hervorgebrachten Aufgaben also erfolgreich lösen kann, sprich kompetent handelt.

Was aber heißt ,korrekt` sprechen können? Knoblauch (2010) weist in seiner begriffsgeschichtlichen Analyse von ,Kompetenz‘ auf die Unterscheidung von Chomsky (1992) zwischen Performanz und Kompetenz hin. Allerdings, so Knoblauch (2010, S. 241), ist die Performanz nicht einfach das Ergebnis von Kompetenz, sondern eine ,abweichende und unvollständige Verwirklichung des idealen Regelwerks“. Kompetenz hat bei Chomsky also einen idealen, reinen Charakter, „die die Performanz des Realen nie erfüllt“ (Knoblauch 2010, S. 241). In diesem Sinne ist die Performanz und somit das ,korrekte" Sprechen immer schon ,fehlerhaft". (Sprach)Kompetenz und (Sprach)Performanz bleibt jedoch auch in dem Sinne ,unvollständig', als dass es das Regelsystem und die regelgeleitete Performanz nicht gibt. Die Regeln sind eben ,keine abstrakten Regeln der Sprache, sondern Regeln des Sprechens in sozialen Situationen“ (Knoblauch 2010, S. 243). Das bedeutet, dass der soziale Kontext bedingt, was angemessenes und damit korrekt Sprechen bedeutet, weshalb dann auch , die fehlerhafte Verwendung der Sprache durchaus regelkonform“ sein kann (Knoblauch 2010, S. 243), bspw. in einem Jugendslang. Es geht also immer darum, was ,,in eben diesem sozialen Zusammenhang als angemessen und korrekt angesehen wird und häufig [als; T.B.] eine Voraussetzung für die 
Zugehörigkeit zur entsprechenden sozialen Gruppe gelten kann“ (Knoblauch 2010, S. 243).

Wenn eine so interpretierte Kompetenz das individuelle „Vermögen zum angemessenen Handeln“ (Knoblauch 2010, S. 247) darstellt, verweist der Terminus ,angemessen“"gleichzeitig auf die konstitutiv kollektive Dimension des individuellen Vermögens. Kompetenz steht somit immer in einem Spannungsverhältnis, in einer Relation zwischen individueller Disposition und kollektiver Rahmung.

\subsection{Konzeption von Praxiskompetenz}

Auf dieses Spannungsverhältnis - sowie auf die weiter oben dargestellte Idee eines impliziten Wissens - verweist der Term der Praxiskompetenz, welcher wesentlich auf dem Konzept des Habitus (Bourdieu 1979) bzw. der Handlungsorientierung (Nohl et al. 2015) aufbaut.

Der Habitus ist „sozialisierte Subjektivität“ (Bourdieu und Wacquant 2013, S. 159) und zeigt sich in „Gestalt der geistigen und körperlichen Wahrnehmungs-, Bewertungs- und Handlungsschemata in den individuellen Körpern“ (Bourdieu und Wacquant 2013, S. 37). Er ist als ,,strukturierte Struktur[]“ (Bourdieu 1987, S. 98) einerseits das Produkt des gesellschaftlichen bzw. praktischen Kontexts seiner Hervorbringung. Andererseits ist er als Inkorporation stark an den Akteur, die Akteurin gebunden, was auch bedeutet, dass ,das Individuelle und selbst das Persönliche, Subjektive, etwas Gesellschaftliches ist, etwas Kollektives“ (Bourdieu und Wacquant 2013, S. 159). Dabei bleibt der Habitus wandelbar, in ihm ist immer „schon eine gewisse Modifikations- und Transformationsfähigkeit angelegt“ (ElMafaalani 2017, S. 122).

Als „strukturierende Struktur[]“ (Bourdieu 1987, S. 98) ist der Habitus ein generatives Prinzip, das im Sinne seiner eigenen Geschichte generiert - und in diesem Sinne eine Kompetenz. Denn der Habitus kann unbegrenzt viele Handlungen hervorbringen, die aber ,innerhalb der Grenzen der besonderen Bedingungen seiner eigenen Hervorbringung liegen“ (Bourdieu 1987, S. 102). Er ist ein ,,sozial konstituiertes System [...], das durch Praxis erworben wird und konstant auf praktische Funktionen“" ausgerichtet ist (Bourdieu und Wacquant 2013, S. 154) - und somit Praxiskompetenz.

Nun spricht Bourdieu selbst meist von ,dem ‘ Habitus, räumt aber prinzipiell die Möglichkeit eines „doppelten Habitus“ (Bourdieu 2015, S. 193) ein. Es muss also betont werden, dass der Habitus ,,keine völlig homogene Struktur bildet, sondern selbst u. U. agonal angelegt ist [...], so ist sogar denkbar, dass sich im Habitus eines Akteurs einander widersprechende oder entgegenstehende" Strukturen finden lassen (Nohl et al. 2015, S. 165). Darauf verweist letztlich auch der Begriff der „Handlungsorientierung“ (Nohl et al. 2015). Handlungsorientierung knüpft terminologisch an den im Rahmen der Dokumentarischen Methode üblichen Begriff des Orientierungsrahmens an, welcher auf den Habitus verweist (Bohnsack 2011, S. 132), bezieht sich jedoch nicht auf ein ganzheitliches Konstrukt - eben den Habitus sondern auf unterhalb dieses ganzheitlichen Konstrukts liegende, gegenstandsbezogene ,Habituierungen“. Handlungsorientierungen sind demnach „Sedimentierungen von Erfahrungs- und Bedeutungsstrukuren“ (Nohl et al. 2015, S. 217), die zwar auf 
einen Gegenstand, also einen Ausschnitt von Welt bezogen sind, allerdings situationsübergreifend auf der Ebene der Akteurinnen und Akteure wirken. Es geht um „modi operandi, mit denen Themen und Problemstellungen des Lebens bewältigt werden“" (Nohl et al. 2015, S. 217). ${ }^{2}$

Geht man davon aus, dass in beruflichen Settings niemals die Welt als Ganzes, sondern immer nur ein Ausschnitt von Welt, ,thematisiert' wird, dann sind es vornehmlich Handlungsorientierungen, welche die individuelle berufliche Kompetenz auf kollektiv-impliziter Ebene ausmachen, sofern sie zum Feld bzw. zu den in der CoP verhandelten Bedeutungsstrukturen passen. Zu einer Kompetenz bzw. zu Praxiskompetenz werden Handlungsorientierungen also erst in ihrer je konkreten Relation zum Feld.

\subsection{Lehrkräftekooperation und Praxiskompetenz}

Versteht man Lehrkräftekooperation als soziale Praxis (CoP), in der kollektiv-implizites Wissen (re)produziert wird, und entsprechend individuelle Kompetenz als Handlungsorientierung(en) und damit als (zumindest zum Teil) durch dieses kollektiv-implizite Wissen geprägt, welche gleichzeitig erst im sozialen Kontext ,Gültigkeit' gewinnt (Praxiskompetenz), dann lassen sich damit zum einen die eingangs widersprüchlichen Befunde zu Lehrkräftekooperation theoretisch fassen und auch ggf. empirisch nachzeichnen, also letztlich begründen, warum Kooperation funktionieren kann, aber scheinbar nicht muss. Zum anderen lässt sich der Modus der Kompetenzentwicklung durch Lehrkräftekooperation neu denken und begreifen eben als ein gegenstandsbezogenes Umlernen habitueller Strukturen (Nohl et al. 2015) und nicht mehr ein Hinzu-Lernen von Wissen. Gleichzeitig lässt sich an bestehende (psychologische) Kompetenzvorstellungen (s. oben) anknüpfen, da auch Praxiskompetenzen erlernt werden, bereichsspezifisch (auf einen Gegenstand von Welt bezogen) und funktional (sie generiert die zur Praxis passenden Handlungen) sind. Die Frage, die sich nun stellt, ist wie sich Lernsituationen, verstanden als Relation zwischen Community und Akteurin bzw. Akteur, gestalten können?

\section{Methodik und Datengrundlage}

Zur Beantwortung der Frage nach der Relation zwischen Akteurin bzw. Akteur sowie Community wurden videographierte Sitzungen von Lehrkräftekooperationsteams insgesamt drei Teams bei mindestens 4 Sitzungen pro Team - sowie elf berufsbiographisch narrative Interviews mit den an diesen Teams beteiligten Lehrerinnen

\footnotetext{
2 Die Idee, den Habitus kleinschrittiger zu denken, findet sich in vielen Ansätzen, wenn auch unter anderen Begriffen. Bremer und Lange-Vester (2014, S. 69) bspw. sprechen in Bezug auf bestimmte Gegenstände von einem „,Zug ' des Habitus“, Helsper (2018, S. 120) spricht von „Differenzierungen des Habitusbegriffs" und unterscheidet zwischen dem primären, dem individuellen und dem feldspezifischen Habitus als „Teilhabitusformen“ in Bezug auf Lehrkräfte. Auch Bohnsack (2020, S. 101) - der dem Konzept der Handlungsorientierung eher skeptisch gegenüber steht (vgl. Bohnsack et al. 2019) (kritisch dazu Bloh 2021, S. 131 ff.) - spricht von bestimmten Erfahrungsräumen, ,welche grundsätzlich lediglich spezifische Bereiche des Individuums, also einen spezifischen Habitus“ betreffen.
} 
erhoben und mittels der Dokumentarischen Methode ausgewertet. In diesem Artikel beschränkt sich die Darstellung der Analyse auf eines der erhobenen Interviews (vgl. zur Interpretation aller Daten Bloh 2021).

Allgemein zeichnet sich die Dokumentarische Methode durch einen Fokus auf implizites, nicht explizierbares und daher eben zu rekonstruierendes Wissen aus. Dieses handlungspraktische Wissen, welches sich der Explikation entzieht, kann im Kontext der Dokumentarischen Methode sowie im Kontext der hier dargelegten Überlegungen auch als „Praktische[r] Sinn“ (Bourdieu und Wacquant 2013, S. 43) bezeichnet werden (Nohl 2017, S. 6).

Forschungspraktisch vollzieht sich die Rekonstruktion dieses Wissens in zwei Arbeitsschritten: der formulierenden und der reflektierenden Interpretation (Nohl 2017). In der formulierenden Interpretation geht es um die Frage, was geschieht, was sich beobachten lässt. Dies dient vornehmlich dazu, sich als Forschende(r), gegenüber dem Text fremd zu machen" (Nohl 2017, S. 31) und zu erkennen, dass bereits die Was-Ebene einer Interpretation bedarf. Auf Basis der formulierenden erfolgt die reflektierende Interpretation, in der es um das Wie, also welcher modus operandi der Handlung zugrunde liegt. Es geht um die „Rekonstruktion und Explikation des Rahmens, innerhalb dessen das Thema abgehandelt wird“ (Bohnsack 2008, S. 135). Im Zuge der reflektierenden Interpretation wird dabei ,konsequent vergleichend“ (Nohl 2017, S. 7) vorgegangen. Der Vergleich erfolgt dabei innerhalb eines Falles (verschiedene Themen innerhalb desselben Falls) und zwischen den Fällen (dieselben Themen in verschiedenen Fällen). Dem Vergleich kommt sowohl eine ,erkenntnisgenerierende“, als auch „,eine erkenntniskontrollierende Funktion“ (Nohl 2013, S. 15) zu.

„Über die Zwecke der Validierung hinaus dient die komparative Sequenzanalyse auch der Generierung mehrdimensionaler Typologien“ (Nohl 2017, S. 41). Hierbei wird ,klassisch` zwischen einer sinn- und einer soziogenetischen Typenbildung unterschieden, wobei es auch neuere Formen und Ideen der Typenbildung gibt (Nohl 2013, 2019). In der hier vorliegenden Untersuchung wurden Relationslogiken - also Relationen zwischen Akteurin bzw. Akteur und Community - v.a. auf Basis der erhobenen Interviews typisiert.

\section{Der Fall Frau Vogt - Kooperationsbedingte Entwicklungsprozesse von Praxiskompetenz}

Die gefundenen und typisierten Relationslogiken lassen sich in allen Fällen rekonstruieren und werden an dieser Stelle exemplarisch am Fall Frau Vogt dargestellt. Frau Vogt ist zum Zeitpunkt des Interviews 29 Jahre alt. Sie ist seit drei Monaten Lehrerin für Sonderpädagogik an der Schule Ka und arbeitet seitdem auch im Team $\mathrm{Ka}$ (einem der videographierten Teams) mit. Letztlich durchläuft Frau Vogt drei Einfindungsprozesse in unterschiedliche Communities of Practice von Lehrkräften. Im Sauerland, in Düren und an der Schule Ka. Während der erste Einfindungsprozess im Sauerland gescheitert ist und abgebrochen wird (Nicht-Passung), ist der zweite in Düren gelungen (Entfaltung). Der dritte ist zum Zeitpunkt des Interviews im Gange (Herausforderung). Zu ihrer ersten Schule berichtet sie Folgendes (Abb. 1). 
180 da, so ca., 9 bis 12 Jahre um da auch anerkannt zu werden; um akzeptiert zu werden dass man da jetzt einfach auch lebt; (.) $m$ - ich mach nur zwei Jahre Referendariat war klar dass mich da keine@akzeptieren würde;@ (.) es warnicht (.) schön da drüben; es war mm-da war man auch unheimlich alleine, die hatten alle ihre eigenen Familien; das war auch ein Kollegium was älter war, an dieser Schule; (.) die brauchten mich nicht; keiner war auf mich angewiesen, ich war die einzige Referendarin; und eh keiner kannte mich; alle waren alt. (2) ja irgendwie ungünstige Bedingungen (.) einfach. blöd gelaufen. (.) das hab ich dann ein Jahr mitgemacht; und, (.) hab mich im Grunde nur noch in die Arbeit reingestürzt, “

Abb. 1 Transkriptausschnitt 1: LK02-Ka-Vogt; Beginn im Sauerland

$\mathrm{Zu}$ ihrer Zeit im Sauerland erzählt Frau Vogt vor allem davon, dass sie keinen sozialen Anschluss gefunden hat und sich als Folge ,nur noch in die Arbeit reingestürzt“ (Z. 187) hat. Das ,die brauchten mich nicht“ (Z. 184) verweist auf eine berufspraktische Isolation und damit auf einen fehlenden Zugang zu einer $\mathrm{CoP}$. Ob der Zugang zur CoP verwehrt bleibt, weil diese - wie Frau Vogt selbst als unumstößlich konstruiert (Z. $181 \mathrm{f}$.) - Frau Vogt ausschließt, kann nicht geklärt werden. Ebenfalls denkbar wäre, dass die Community selbst über einen Modus Operandi verfügt, der eine (enge) Zusammenarbeit ausschließt. In diesem Fall wäre es kein (mehr oder weniger) aktives Ausschließen von Frau Vogt durch die Community, sondern eine Community, die das Bedürfnis von Frau Vogt (scheinbar) nicht abdeckt - die über eine differente Praxis verfügt. Die Relation zwischen der Community of Practice und Frau Vogt wäre so oder so eine Nicht-Passung von Praxen (Relationslogik der Nicht-Passung). Sie kann hier auf habitueller Ebene nicht lernen, da die beiden Praxen ,polar' (Helsper 2018) zueinander stehen. verteilt; und zwar ganz spezielle Komplimente; wie zum Beispiel, ähm (.) wenn er zu ner Frau gesagt hat sie haben (.) das sehr männlich gemacht; zum Beispiel; oder sie sind sehr männlich; oder sie sind wie Herr (.) töktöktöktöktök. (.) da war das n Kompliment; (2) //mhm// und wenn man sich eher weiblich gegeben hat; oder, eher (.) so defensiver war, oder beschwichtigend; (2) das war für ihn, (.) schlecht; (.) //mhm// das war für ihn wirklich das hat er auch rückgemeldet; dass er das nicht sehen möchte; (.) das Ganze wurde nochmal extra unterstützt von der Konrektorin (2) das äh sie im Grunde dass was verlangt wird; nämlich dieses harte; dieses dominante; autoritäre; das hat die nochmal extra angestachelt; (.) sie müssen noch härter werden, sie müssen noch strenger werden, (2) also das wurde da wirklich gepuscht; (.) also (2) und ich hab mich äh sehr (2) angestrengt, diesen (.) Bedingungen einfach irgendwie, auch äh zu entsprechen; dass ich das alles auch schaffe; ja ich bin männlich, ich bin hart; ich kann das; und dies; und das. “

Abb. 2 Transkriptausschnitt 2: LK02-Ka-Vogt; Komplimente des Schulleiters 
Nachdem Frau Vogt es durch einen Autounfall und die damit verbundene Bewilligung ihres Versetzungsantrags geschafft hat, die Schule zu verlassen, kommt sie wieder ,rein in die Zivilisation“ (Z. 193) - an eine Schule in Düren. Diese Schule zeichnet sich stark durch Gewalt aus (Z. $300 \mathrm{ff}$.), der mit Disziplinierung begegnet wird - einer Praxis, die Frau Vogt näher liegt (Abb. 2).

In dieser Passage dokumentiert sich zweierlei. Zum einen wird deutlich, dass die konkrete Praxis bzw. das ,richtige“ Verhalten kollektiv bedingt sind. An der Schule werden, hier repräsentiert durch den Schulleiter und die Konrektorin, in anderen Passagen durch das Kollegium, gewisse Handlungen positiv (,männlich“), andere negativ (,weiblich“) bewertet. Zum anderen wird deutlich, dass Frau Vogt diese kollektive Sichtweise für sich annimmt, indem sie ihr entsprechen möchte. Dies sind die „Bedingungen“ (Z. 323), eben das, was erfüllt sein muss, um an der Praxis teilzuhaben und Wertschätzung zu bekommen bzw. um ,gut‘ zu handeln.

Die Praxis des dominanten Auftretens bzw. des Disziplinierens ist dabei in besonderer Weise anschlussfähig an die berufsbiographische Orientierung von Frau Vogt. Disziplin ist für Frau Vogt nicht nur in Bezug auf sich selbst bedeutsam, ${ }^{3}$ sondern wird hier auch von den Schülerinnen und Schülern verlangt. Im Rahmen der kollektiven Praxis des dominanten Auftretens kann sie sich frei entfalten (Relationslogik der Entfaltung). Ein Lernen auf habitueller Ebene ist hier nicht nötig.

Frau Vogt entfaltet in ihrer Phase in Düren Denk-, Wahrnehmungs- und Handlungsschemata in Bezug auf ihre Praxis. Sie entfaltet Handlungsorientierungen in Bezug auf Gegenstände von Welt. Sie lebt die Praxis des dominanten Auftretens. Diese werden mit dem Wechsel an die Schule Ka brüchig, was sich unter anderem in der rückblickenden Bewertung dokumentiert (Abb. 3).

Die damalige Sicht- und Arbeitsweise erscheint ihr mittlerweile als ,verquer“ (Z. 332), also als nicht normal, als merkwürdig. Dass sie die Dinge damals selbst so gesehen hat, kann sie heute nicht mehr glauben, sie echauffiert (,hallo?“) sich sogar darüber. Das zeigt auch, bzw. macht es notwendig, eine theoretische Erklärung, die „Betriebsblindheit“ (Z. 333) anzuführen. Mit Betriebsblindheit ist gemeint, dass man gewisse Dinge ,von Innen“ nicht sehen kann, da man eben mit einer bestimmten Brille auf die Welt schaut, eben mit der des Betriebes bzw. der der Community. Erst wenn man diese Brille ablegt, sieht man die Dinge anders - eben mit einer neuen Brille.

Dass Frau Vogt Dinge so kritisiert, die sie zuvor in ihrer Betriebsblindheit fraglos hingenommen hat, verweist auf einen Bruch in den Denk-, Handlungs- und Wahrnehmungsschemata in Bezug auf die schulische Arbeit (Bruch der Praxiskompetenz).

332 es waren vor- (.) hallo? (2) es war einfach $n$ (.) vollkommen, (3) verqueres Denken irgendwie;

333 Betriebsblindheit; “

Abb. 3 Transkriptausschnitt 3: LK02-Ka-Vogt; Verqueres Denken

\footnotetext{
3 Aus Frau Vogts (berufs)biographischen Erzählungen lässt sich eine für sie zentrale Logik rekonstruieren, die des Alleine-Schaffens. Frau Vogt nimmt für sich stets auch Unbequemlichkeiten in Kauf, um schwierige Situationen zu bewältigen - sie diszipliniert sich und verlangt dies auch von anderen (dazu ausührlich Bloh 2021). Bremer und Lange-Vester (2014, S. 74) verweisen auf ein ähnliches Phänomen, dass Bildungsaufsteigerinnen und -aufsteiger ihre eigene gezeigte „Leistung und Anstrengungsbereitschaft“ als Lehrkraft auch von ihren Schülerinnen und Schülern verlangen.
} 
204 bestimmte Vorstellungen und ich kann manchmal sagen Halt Stopp ne also jetzt (.) das sind

205 Grundschulkinder sie hat vorher mit größeren älteren gearbeitet, ne? //mhm// die lernen das

206 alles jetzt noch nicht so hart,"

Abb. 4 Transkriptausschnitt 4: LK05-Ka-Schröder; Grundschulkinder lernen anders

Die damaligen Sichtweisen erscheinen nicht mehr angemessen. Diese Hinterfragung des Alten ist dabei erst möglich, indem etwas Neues kommt. Das Alte (die Praxis in Düren) wird erst durch das Neue (die Praxis an der Schule Ka) zu etwas Altem und dadurch zu etwas Hinterfragbarem. Dieses Hinterfragen geschieht dabei aber nicht individuell, sondern auf Basis wiederum kollektiver Ansprüche, wie sich exemplarisch an folgender Passage von Frau Schröder verdeutlichen lässt (Abb. 4).

In dieser Passage erzählt Frau Schröder - die wie Frau Vogt Teil des Kooperationsteams „Ka“ ist - von der Zusammenarbeit mit Frau Vogt. ${ }^{4}$ Liegt der Blick auf die Kinder zwar im Alter („Grundschulkinder“; Z. 205) und damit der Entwicklungsebene begründet, reicht dies für Frau Vogt scheinbar nicht aus, um ,adäquate“ Handlungen im Sinne der Community auszuführen - Frau Vogt handelt zunächst immer noch hart und dominant, obwohl die Kinder jünger sind. Die veränderten situativen Bedingungen (jüngere Kinder, anderes Umfeld) könnten zwar ebenso eine Rolle spielen, entscheidend ist aber die kollektive Angemessenheit der Handlung, also die anderen Lehrkräfte, hier verkörpert durch Frau Schröder. Es bedarf des Einschreitens einer anderen Lehrerin, die die entsprechende Handlung bzw. die „Vorstellungen“ (Z. 204) kritisiert (,hart“; Z. 206) und Frau Vogt zum Ändern, zumindest zum Aufhören („Halt Stopp“; Z. 204) auffordert.

Die Art und Weise der Handlungen von Frau Vogt passen nicht mehr, sind nicht mehr adäquat. Die Sanktionierung durch eine andere Lehrerin markiert die Handlung als nicht feldkonform, als Regelverletzung - wodurch ihr feld- bzw. communityspezifisch eine Bedeutung zugeschrieben wird. Ob und inwieweit Frau Vogt die neue Handlungsorientierung hier übernimmt, kann nicht genau gesagt werden. Die Distanzierung von ihrer vorherigen Praxis, die Anerkennung und Wertung der jetzigen Sicht als ,gesünder“ (Z. 360), sind jedoch Hinweise darauf, dass die Sanktionierung der nicht feldkonformen Ausübung von Handlungen einen Bruch der Praxiskompetenz (mit)erzeugt haben - und Frau Vogt hier nun lernt, im Sinne der neuen Community zu handeln. In diesem Sinne wird Frau Vogt durch die (neue) Praxis des Teams Ka herausgefordert (Relationslogik der Herausforderung) und zu einem Umlernen angeregt. Es zeigt sich also eine Veränderung bei Frau Vogt, angeregt durch die Sichtweise(n) in Team Ka - es zeigt sich der Beginn einer Praxiskompetenzentwicklung durch Lehrkräftekooperationsprozesse. ${ }^{5}$

\footnotetext{
4 Auch aus den Interviews der anderen Teammitglieder sowie aus den Rekonstruktionen der Teamsitzungen lassen sich ähnliche ,Lernaufforderungen“ an Frau Vogt rekonstruieren (vgl. dazu genauer Bloh 2021; Abschn. 8.1 und 9.1).

5 Inwieweit die beiden anderen Einfindungsprozesse auf konkrete Kooperationsteams oder auf größere CoPs (bspw. das Kollegium) im Sinne einer (dann jedoch sehr homogenen) Schulkultur (Helsper 2008) zurückgeführt werden können, kann aus dem Material nicht abschließend geklärt werden - auch wenn sich Indizien für kleinere CoPs i. S. v. Kooperationsteams finden lassen.
} 


\section{Fazit und Ausblick}

Die drei hier dargestellten - und auch in anderen Fällen rekonstruierten (vgl. Bloh 2021, S. 220 ff.) - Relationslogiken der Nicht-Passung, Entfaltung und Herausforderung verweisen auf eine spezifische Form des Kontakts zwischen Akteurin bzw. Akteur einerseits, und Community of Practice andererseits. Es wird deutlich, dass Kompetenz sowie die Notwendigkeit zur Kompetenzentwicklung nicht ausschließlich von der Aufgabe (bspw. dem Umgang mit Kindern) gedacht werden kann, wie in ,klassischen“ Kompetenzkonstrukten (Klieme und Hartig 2007), sondern sich auch (und m. E. wesentlich) aus dem sozialen Kontext ergibt - und damit immer relational zu einer sozialen Praxis ist, worauf der Begriff Praxiskompetenz verweist. Die gegenstandsbezogenen Denk-, Wahrnehmungs- und Handlungsschemata können in dem Moment brüchig werden, in dem sie durch einen Wechsel der Community, also einem Wechsel des sozialen Kontexts, herausgefordert werden - während Kompetenz im erziehungswissenschaftlich-psychologischen Sinne (Klieme und Hartig 2007) jenseits dieses Kontexts funktioniert. Passen sie dagegen nicht, oder entfalten sich in der Praxis, ist ein ,Lernen“ auf habitueller Ebene (Nohl et al. 2015) nicht möglich bzw. unnötig. Wie bedeutsam Praxiskompetenz für die berufliche Praxis ist, deutet der Fall Frau Vogt an, da hier unter anderem die gesamte Sichtweise auf und der Umgang mit Kindern ins Wanken gerät und sich transformiert. Die alte, zuvor selbstverständliche, Sichtweise wird vor dem Hintergrund der neuen Perspektive fragwürdig und kritisch.

Das Konstrukt Lehrkräftekooperation (Fussangel und Gräsel 2014) gewinnt vor diesem Hintergrund eine gänzlich andere Bedeutung, da es als soziale Praxis weniger ,steuerbar', weniger als Instrument nutzbar ist, sondern eine Eigenlogik entwickelt. Vor dem Hintergrund dieser Eigenlogik sind dann einerseits die eingangs erwähnten widersprüchlichen Befunde erklärbar, andererseits muss einem allzu positivem Blick, der die Bedeutung der Kooperation für die Professionalität der beteiligten Lehrkräfte ausschließlich positiv einschätzt, m. E. skeptisch gegenüber gestanden werden.

Geht man davon aus, dass Praxiskompetenz bedeutsam für Handlungen ist, dann stellt sich weiterhin und notgedrungen die Frage nach der Bewertung von Praxiskompetenzen. Während einerseits Praxiskompetenz aus der Praxis selbst bewertet wird, indem sie dort eben als Kompetenz anerkannt wird, ist diese Perspektive, andererseits, in Bezug auf Qualitätsfragen (,guter' Unterricht; ,positive' Schulentwicklung) unbefriedigend. Eine praxeologische Evaluation(sforschung) muss demnach Mittel und Wege finden, die eine Bewertung jenseits praxisimmanenter Normierungen zulässt, ohne dabei den praktischen Blick zu verlieren. Dazu müssen „Bezugssysteme und Relevanzkriterien“ (Lüders und Haubrich 2003, S. 322) klar umrissen, dennoch aber praktisch formuliert werden. Hierzu kann die Dokumentarische Evaluationsforschung (Bohnsack und Nentwig-Gesemann 2020) einen Ansatz bieten, muss jedoch hinsichtlich der Explikation eben jener Bezugssysteme und Relevanzkriterien weiter ausgearbeitet werden.

Funding Open Access funding enabled and organized by Projekt DEAL. 
Open Access Dieser Artikel wird unter der Creative Commons Namensnennung 4.0 International Lizenz veröffentlicht, welche die Nutzung, Vervielfältigung, Bearbeitung, Verbreitung und Wiedergabe in jeglichem Medium und Format erlaubt, sofern Sie den/die ursprünglichen Autor(en) und die Quelle ordnungsgemäß nennen, einen Link zur Creative Commons Lizenz beifügen und angeben, ob Änderungen vorgenommen wurden.

Die in diesem Artikel enthaltenen Bilder und sonstiges Drittmaterial unterliegen ebenfalls der genannten Creative Commons Lizenz, sofern sich aus der Abbildungslegende nichts anderes ergibt. Sofern das betreffende Material nicht unter der genannten Creative Commons Lizenz steht und die betreffende Handlung nicht nach gesetzlichen Vorschriften erlaubt ist, ist für die oben aufgeführten Weiterverwendungen des Materials die Einwilligung des jeweiligen Rechteinhabers einzuholen.

Weitere Details zur Lizenz entnehmen Sie bitte der Lizenzinformation auf http://creativecommons.org/ licenses/by/4.0/deed.de.

\section{Literatur}

Achinstein, B. (2002). Conflict amid community: the micropolitics of teacher collaboration. Teachers College Record, 104(3), 421-455.

Alkemeyer, T., \& Buschmann, N. (2017). Befähigen. Praxistheoretische Überlegungen zur Subjektivierung von Mitspielfähigkeit. In M. Rieger-Ladich \& C. Grabau (Hrsg.), Pierre Bourdieu: Pädagogische Lektüren (S. 271-297). Wiesbaden: Springer VS.

Asbrand, B. (2008). Wie erwerben Jugendliche Wissen und Handlungsorientierung in der Weltgesellschaft. Globales Lernen aus der Perspektive qualitativ-rekonstruktiver Forschung. Zeitschrift für Internationale Bildungsforschung und Entwicklungspädagogik, 31(1), 4-8.

Bloh, T. (2021). Kooperation und Praxiskompetenz. Eine praxeologische Perspektive auf kooperationsbedingte Kompetenzentwicklung von Lehrkräften. Wiesbaden: Springer VS.

Bloh, T., \& Bloh, B. (2016). Lehrerkooperation als Community of Practice - Zur Bedeutung kollektivimpliziter Wissensbestände für eine kooperationsbedingte Kompetenzentwicklung. Journal for Educational Research Online, 8(3), 207-230.

Bloh, T., \& Bloh, B. (2020). Professionelle Lerngemeinschaften als Entwicklungsinstrument - Eine kritische Reflexion. In K. Kansteiner, C. Stamann, C. Buhren \& P. Theurl (Hrsg.), Professionelle Lerngemeinschaften als Entwicklungsinstrument im Bildungswesen (S. 49-62).

Bohnsack, R. (2008). Rekonstruktive Sozialforschung. Einführung in qualitative Methoden (7. Aufl.). Opladen: Barbara Budrich/UTB.

Bohnsack, R. (2011). Orientierungsmuster. In R. Bohnsack, W. Marotzki \& M. Meuser (Hrsg.), Hauptbegriffe Qualitativer Sozialforschung (3. Aufl. S. 132-133). Opladen: Barbara Budrich.

Bohnsack, R. (2017). Praxeologische Wissenssoziologie. Opladen: Barbara Budrich.

Bohnsack, R. (2020). Professionalisierung in praxeologischer Perspektive. Zur Eigenlogik der Praxis in Lehramt, Sozialer Arbeit und Frühpädagogik. Opladen: Barbara Budrich/UTB.

Bohnsack, R., \& Nentwig-Gesemann, I. (Hrsg.). (2020). Dokumentarische Evaluationsforschung. Theoretische Grundlagen und Beispiele aus der Praxis. Bd. 2. Opladen: Barbara Budrich.

Bohnsack, R., Nentwig-Gesemann, I., \& Hoffmann, N.F. (2019). Typenbildung und Dokumentarische Methode. In S. Amling, A. Geimer, A.-C. Schondelmayer, K. Stützel \& S. Thomsen (Hrsg.), Jahrbuch Dokumentarische Methode. Heft 1/2019 (S. 17-50). Berlin: centrum für qualitative evaluations- und sozialforschung e. V. (ces).

Bonsen, M., \& Rolff, H.-G. (2006). Professionelle Lerngemeinschaften von Lehrerinnen und Lehrern. Zeitschrift für Pädagogik, 52(2), 167-184.

Bourdieu, P. (1979). Entwurf einer Theorie der Praxis. Frankfurt a.M.: Suhrkamp.

Bourdieu, P. (1987). Sozialer Sinn. Frankfurt a.M.: Suhrkamp.

Bourdieu, P. (1992). Rede und Antwort. Frankfurt a.M.: Suhrkamp.

Bourdieu, P. (2015). Praktische Vernunft. Zur Theorie des Handelns (9. Aufl.). Frankfurt a.M.: Suhrkamp.

Bourdieu, P., \& Wacquant, L. J. D. (2013). Reflexive Anthropologie (3. Aufl.). Frankfurt a.M.: Suhrkamp.

Bremer, H., \& Lange-Vester, A. (2014). Die Pluralität der Habitus- und Milieuformen bei Lernenden und Lehrenden. Theoretische und methodologische Überlegungen zum Verhältnis von Habitus und sozialen Raum. In W. Helsper, R.-T. Kramer \& S. Thiersch (Hrsg.), Schülerhabitus. Theoretische und empirische Analysen zum Bourdieuschen Theorem der kulturellen Passung (S. 56-81). Wiesbaden: Springer VS. 
Chomsky, N. (1992). Aspekte der Syntax-Theorie (3. Aufl.). Frankfurt a.M.: Suhrkamp.

El-Mafaalani, A. (2017). Transformation des Habitus. Praxeologische Zugänge zu sozialer Ungleichheit und Mobilität. In M. Rieger-Ladich \& C. Grabau (Hrsg.), Pierre Bourdieu: Pädagogische Lektüren (S. 103-127). Wiesbaden: Springer VS.

Eraut, M. (2000). Non-formal learning and tacit knowledge in professional work. British Journal of Educational Psychology, 70(1), 113-136.

Fussangel, K., \& Gräsel, C. (2014). Forschung zur Kooperation im Lehrerberuf. In E. Terhart, H. Bennewitz \& M. Rothland (Hrsg.), Handbuch der Forschung zum Lehrerberuf(Bd. 2, S. 846-864). Münster: Waxmann.

Fussangel, K. (2008). Subjektive Theorien von Lehrkräften zur Kooperation. Eine Analyse der Zusammenarbeit von Lehrerinnen und Lehrern in Lerngemeinschaften. http://nbn-resolving.de/urn/resolver.pl? urn=urn\%3Anbn\%3Ade\%3Ahbz\%3A468-20080475. Zugegriffen: 8. Sept. 2015.

Gräsel, C., Pröbstel, C., Freienberg, J., \& Parchmann, I. (2006). Anregungen zur Kooperation von Lehrkräften im Rahmen von Fortbildungen. In M. Prenzel \& L. Allolio-Näcke (Hrsg.), Untersuchungen zur Bildungsqualität von Schule (S. 310-329). Münster: Waxmann.

Grossmann, P., Wineburg, S., \& Woolworth, S. (2001). Toward a theory of teacher community. Teachers College Record, 103(6), 942-1012.

Gruber, H. (1999). Erfahrung als Grundlage kompetenten Handelns. Göttingen: Huber.

Halbheer, U., \& Kunz, A. (2009). Mehr Schulqualität dank Kooperation? Eine quantitativ-qualitative Beschreibung zur Kooperation zwischen Lehrpersonen. In K. Maag Merki (Hrsg.), Kooperation und Netzwerkbildung. Strategien der Qualitätsentwicklung in Schulen (S. 66-77). Stuttgart: Klett.

Helsper, W. (2008). Schulkulturen - die Schule als symbolische Sinnordnung. Zeitschrift für Pädagogik, 54(1), 63-80.

Helsper, W. (2018). Lehrerhabitus. Lehrer zwischen Herkunft, Milieu und Profession. In A. Paseka, M. Keller-Schneider \& A. Combe (Hrsg.), Ungewissheit als Herausforderung für pädagogisches Handeln (S. 105-140). Wiesbaden: Springer VS.

Hodkinson, H., \& Hodkinson, P. (2004). Rethinking the concept of community of practice in relation to schoolteachers' workplace learning. International Journal of Training and Development, 8(1), 21-31.

Horn, I. S. (2005). Learning on the job: a situated account of teacher learning in high school mathematics departments. Cognition and Instruction, 23(2), 207-236.

Horn, I. S. (2015). Teachers learning together: pedagogical reasoning in mathematics teachers' collaborative conversation. In S.J. Cho (Hrsg.), Selected regular Lecturesfrom the 12th internationalselected regular lectures from the 12th international congress on mathematical education (S. 333-342). Heidelberg: Springer.

Horn, I.S., \& Little, J.W. (2010). Attending problems of practice: routines and resources for professional learning in teachers' workplace interactions. American Educational Research Journal, 47(1), 181-217.

Järvinen, H., Manitius, V., \& Otto, J. (2012). Arbeiten in schulischen Netzwerken - Das Beispiel Schulen im Team. In S. G. Huber \& F. Ahlgrimm (Hrsg.), Kooperation. Aktuelle Forschung zur Kooperation in und zwischen Schulen sowie mit anderen Partnern (S. 262-282). Münster: Waxmann.

Kansteiner, K., Stamann, C., Buhren, C., \& Theurl, P. (Hrsg.). (2020). Professionelle Lerngemeinschaften als Entwicklungsinstrument im Bildungswesen

Klieme, E., \& Hartig, J. (2007). Kompetenzkonzepte in den Sozialwissenschaften und im erziehungswissenschaftlichen Diskurs. Zeitschrift für Erziehungswissenschaft, 10(8), 11-29.

Knoblauch, H. (2010). Von der Kompetenz zur Performanz. Wissenssoziologische Aspekte der Kompetenz. In T. Kurtz \& M. Pfadenhauer (Hrsg.), Soziologie der Kompetenz (S. 237-255). Wiesbaden: VS.

Krais, B., \& Gebauer, G. (2014). Habitus (6. Aufl.). Bielefeld: transcript.

Kullmann, H. (2009). Lehrerkooperation an Gymnasien. Eine explorative Untersuchung zu Ausprägungen und Wirkungen am Beispiel des naturwissenschaftlichen Unterrichts. Dissertation an der Universität Duisburg-Essen. http://duepublico.uni-duisburg-essen.de/servlets/DerivateServlet/Derivate22359/Dissertation_Kullmann.pdf. Zugegriffen: 14. Okt. 2012.

Lave, J., \& Wenger, E. (1991). Situated Learning. Legitimate peripheral participation. Cambridge: University Press.

Lomos, C., Hofman, R.H., \& Bosker, R.J. (2011). Professional communities and student achievement-a meta-analysis. School Effectiveness and School Improvement, 22(2), 121-148.

Louis, K.S., Marks, H.M., \& Kruse, S.D. (1996). Teachers' professional community in restructuring schools. American Educational Research Journal, 33(4), 757-798. 
Lüders, C., \& Haubrich, K. (2003). Qualitative Evaluationsforschung. In C. Schweppe (Hrsg.), Qualitative Forschung in der Sozialpädagogik (S. 305-330). Wiesbaden: Springer.

Mannheim, K. (1995). Ideologie und Utopie (8. Aufl.). Frankfurt a.M.: Vittorio Klostermann.

Martens, M. (2010). Implizites Wissen und kompetentes Handeln. Die empirische Rekonstruktion von Kompetenzen historischen Verstehens im Umgang mit Darstellungen von Geschichte. Beihefte zur Zeitschrift für Geschichtsdidaktik, Bd. 1. Göttingen: V \& R Unipress.

Neumann, K. (2013). Mit welchem Auflösungsgrad können Kompetenzen modelliert werden? In welcher Beziehung stehen Modelle zueinander, die Kompetenz in einer Domäne mit unterschiedlichem Auflösungsgrad beschreiben? Zeitschrift für Erziehungswissenschaft, Sonderheft, 18, 34-39.

Neuweg, G. H. (2001). Könnerschaft und implizites Wissen. Zur lehr-lerntheoretischen Bedeutung der Erkenntnis- und Wissenstheorie Michael Polanyis (2. Aufl.). Münster: Waxmann.

Nohl, A.-M. (2013). Relationale Typenbildung und Mehrebenenvergleich. Neue Wege der dokumentarischen Methode. Wiesbaden: Springer VS.

Nohl, A.-M. (2017). Interview und Dokumentarische Methode. Anleitungen für die Forschungspraxis (5. Aufl.). Wiesbaden: Springer VS.

Nohl, A.-M. (2019). Zur Bedeutung der relationalen Typenbildung für die Dokumentarische Methode. In S. Amling, A. Geimer, A.-C. Schondelmayer, K. Stützel \& S. Thomsen (Hrsg.), Jahrbuch Dokumentarische Methode. Heft 1/2019 (S. 51-64). Berlin: centrum für qualitative evaluations- und sozialforschung e. V. (ces).

Nohl, A.-M., von Rosenberg, F., \& Thomsen, S. (2015). Bildung und Lernen im biographischen Kontext. Empirische Typisierungen und praxeologische Reflexionen. Wiesbaden: Springer VS.

Pfadenhauer, M. (2010). Kompetenz als Qualität sozialen Handelns. In T. Kurtz \& M. Pfadenhauer (Hrsg.), Soziologie der Kompetenz (S. 149-172). Wiesbaden: VS.

Polanyi, M. (2016). Implizites Wissen (2. Aufl.). Frankfurt a.M.: Suhrkamp.

Przyborski, A. (2004). Gesprächsanalyse und dokumentarische Methode. Qualitative Auswertung von Gesprächen, Gruppendiskussionen und anderen Diskursen. Wiesbaden: VS.

Richter, D., \& Pant, H. A. (2016). Lehrerkooperation in Deutschland. Eine Studie zu kooperativen Arbeitsbeziehungen bei Lehrkräften der Sekundarstufe I. Gütersloh: Bertelsmann Stiftung.

Rolff, H.-G. (2015). Professionelle Lerngemeinschaften als Königsweg. In H.-G. Rolff (Hrsg.), Handbuch Unterrichtsentwicklung (S. 564-575). Weinheim: Beltz.

Steinert, B., Klieme, E., Maag Merki, K., Döbrich, P., Halbheer, U., \& Kunz, A. (2006). Lehrerkooperation in der Schule: Erfassung, Konzeption, Ergebnisse. Zeitschrift für Pädagogik, 52(2), 185-204.

Trumpa, S., Franz, E.-K., \& Greiten, S. (2016). Forschungsbefunde zur Kooperation von Lehrkräften. Ein narratives Review. Die Deutsche Schule, 108(1), 80-92.

Vangrieken, K., Dochy, F., Raes, E., \& Kyndt, E. (2015). Teacher collaboration: a systematic review. Educational Research Review, 15, 17-40.

Vescio, V., Ross, D., \& Adams, A. (2008). A review of research on the impact of professional learning communities on teaching practice and student learning. Teaching and Teacher Education, 24(1), $80-91$.

Weinert, F.E. (2001). Concept of competence: a conceptual clarification. In D. S. Rychen \& L. H. Salganik (Hrsg.), Defning and selecting key competencies (S. 45-65). Göttingen: Hogrefe \& Huber.

Wenger, E. (2000). Communities of practice and social learning systems. Organization, 7(2), 225-246.

Wilhelm, O., \& Nickolaus, R. (2013). Was grenzt das Kompetenzkonzept von etablierten Kategorien wie Fähigkeit, Fertigkeit oder Intelligenz ab? Zeitschrift für Erziehungswissenschaft, Sonderheft, 18, 23-26.

Windeler, A. (2014). Können und Kompetenzen von Individuen, Organisationen und Netzwerken. Eine praxistheoretische Perspektive. In A. Windeler \& J. Sydow (Hrsg.), Kompetenz. Sozialtheoretische Perspektiven (S. 225-301). Wiesbaden: Springer VS. 